\title{
BALANÇO DE ENERGIA EM VINHEDO DE 'NIAGARA ROSADA’(1)
}

\author{
JOSÉ RICARDO MACEDO PEZZOPANE ${ }^{(2)}$; MÁRIO JOSÉ PEDRO JÚNIOR ${ }^{(3,4)}$
}

\begin{abstract}
RESUMO
O método do balanço de energia foi utilizado para caracterizar a variação horária do saldo de radiação e dos fluxos de calor latente, sensível e no solo, em vinhedo cultivado com a cultivar NiagaraRosada', conduzida no sistema de espaldeira, em Jundiaí (SP). Além disso, foram determinadas as relações entre o saldo de radiação (SR) no vinhedo e a radiação solar global (RG) e a partição da energia disponível ao sistema nos fluxos de calor latente (LE), sensível (H) e no solo (G). Em um dia característico de período seco, o LE representou $44 \%$ do SR e o H, 48\%. Em um dia chuvoso, o LE representou $86 \%$ do SR e o H, 21\%. Em um dia ensolarado, após um período de chuvas, LE e H foram, respectivamente, $68 \%$ e $29 \%$ do SR. O G foi, em média, 5,7\% e 1,3\% do SR para as ruas mantidas capinadas e com forro, respectivamente.

Palavras-chave : videira, saldo de radiação, fluxo de calor latente, fluxo de calor sensível e fluxo de calor no solo.
\end{abstract}

\section{ABSTRACT \\ ENERGY BALANCE ON 'NIAGARA ROSADA’ VINEYARD}

The energy balance method was used to characterize the hourly variation of the net radiation, latent and sensible fluxes and soil heat flux on a mature vineyard grown at Jundiaí, São Paulo, Brazil. The grapevines, cv. Niagara Rosada, in the vineyard were wrapped to trellis wires, creating compact hedgerows $2 \mathrm{~m}$ apart, $1.7 \mathrm{~m}$ height and 0.4 wide, with the foliage $1 \mathrm{~m}$ above the soil surface. Also, the net and incoming radiation relationships and the partioning of the available energy to the system into latent and heat flux, and soil heat flux were determined for the vineyard. During a sunny day (dry period) the latent heat flux was $44 \%$ of the net radiation and the sensible heat flux, $48 \%$. However during a rainy day, the latent heat flux was $86 \%$ of the net radiation and the sensible heat flux, $21 \%$. During a sunny day, after the occurrence of rain, the latent and sensible heat fluxes were, respectively, $68 \%$ and $29 \%$ of the net radiation. The soil heat flux was 5.7 an $1.3 \%$ of the net radiation, for bare soil and mulched rows, respectively.

Key words: grapevine, net radiation, soil heat flux, latent and sensible fluxes.

$\left(^{1}\right)$ Recebido para publicação em 3 de maio e aceito em 23 de novembro de 2002.

( $\left.{ }^{2}\right)$ Pós-Graduando em Física do Ambiente Agrícola - Escola Superior de Agricultura “Luiz de Queiroz"(ESALQ/USP). E-mail: rmpezzo@carpa.ciagri.usp.br. Bolsista FAPESP

$\left({ }^{3}\right)$ Centro de Pesquisa e Desenvolvimento de Ecofisiologia e Biofísica (IAC), Caixa Postal 28, 13001-970 Campinas (SP)

$\left({ }^{4}\right)$ Com bolsa de Produtividade em Pesquisa do CNPq. 


\section{INTRODUÇÃO}

A videira Niagara Rosada é a principal cultivar de uva de mesa plantada no Estado de São Paulo. A maioria dos cultivos é realizada em espaldeira e, para amenizar o problema da exposição do solo difundiu-se entre os viticultores a prática do uso de forro entre as ruas do vinhedo (POMMER et al., 1991).

Esse sistema de produção propicia um microclima especial para o vinhedo, pois a distribuição da radiação solar dentro do dossel das videiras é muito influenciada pelo sistema de condução e tipo de poda verde utilizada pelo viticultor (SMART, 1973), além da influência marcante proporcionada pela utilização do forro como cobertura morta.

A energia utilizada nos processos de transferência de água de uma superfície para a atmosfera, de aquecimento e resfriamento do ar e solo, bem como para a realização do metabolismo das plantas, é proveniente da radiação solar. Portanto, em uma superfície vegetada, é importante o conhecimento da partição da radiação solar nestes processos, para estudos da variação do consumo de água durante o crescimento da cultura (Alves et al., 1998), que influencia nos processos de formação da produção e acúmulo de açúcar (Pommer e PAssos, 1990).

O método de balanço de energia, com base no princípio da conservação de energia, destaca- se na determinação do consumo de água por uma comunidade vegetal. Apesar de suas limitações, tem sido utilizado por muito autores ( VILla Nova et al., 1975; Cunha et al. 1996; Teixeira et al., 1997; Alves et al., 1998; Lopes et al., 2001).

Na cultura da videira, sob outras condições de condução e manejo, esse tipo de estudo foi realizado por Oliver e SENe (1992), na região central da Espanha, HeIlman et. al. (1994) no Texas, onde estes autores verificaram grande contribuição do calor sensível gerado na superfície do solo no balanço de energia e por TeIXEIRA et al. (1997), no Nordeste Brasileiro. Este trabalho teve por objetivo determinar as relações entre a radiação solar global, saldo de radiação e fluxo de calor no solo, e caracterizar a partição do saldo de radiação nos fluxos de calor latente, sensível e calor no solo em videira "Niagara Rosada" conduzida em espaldeira.

\section{MATERIAL E MÉTODOS}

O experimento foi desenvolvido na Estação Experimental de Agronomia de Jundiaí, do Instituto Agronômico, durante o ano agrícola de 1998/99. Os dados foram coletados em videiras cv. 'Niagara Ro- sada' conduzidas em espaldeira, com altura aproximada de 1,5 m, com três fios de arame, em espaçamento de $2 \times 1 \mathrm{~m}$. Como medida de proteção do solo, utilizou-se forro, com capim gordura seco em ruas alternadas na lavoura.

Durante o desenvolvimento da cultura efetuaram-se medições do saldo de radiação, fluxo de calor no solo, gradientes de temperatura seca $(\Delta \mathrm{Ts})$ e úmida $(\Delta \mathrm{Tu})$, com psicrômetros de termopares. O saldo radiômetro (modelo Q7, Rebbs) foi instalado $2 \mathrm{~m}$ acima do dossel da videira, os fluxímetros (Middleton) posicionados a $2 \mathrm{~cm}$ de profundidade em ruas alternadas, amostrando o fluxo de calor no solo nas entrelinhas com solo capinado e forrado com capimgordura seco; instalaram-se os psicrômetros de termopar em microabrigos localizados a $0,4 \mathrm{~m}$ e 1,4 $\mathrm{m}$ acima do topo da cultura. Os dados diários de temperatura máxima, temperatura mínima, precipitação e radiação solar global, utilizados para a caracterização do período de avaliações, foram obtidos no posto meteorológico da Estação Experimental, situado a aproximadamente $150 \mathrm{~m}$ do experimento. As medidas foram efetuadas entre 16/10 e 16/12/98.

As leituras micrometeorológicas do experimento foram realizadas em amostragens a cada cinco segundos e os dados armazenados em um sistema automático de aquisição (modelo CR10X, Campbell Scientific.) programado para realizar médias horárias. A partir dos dados, empregando-se a razão entre o fluxo de calor sensível $(\mathrm{H})$ e latente (IE), proposta por Bowen (equação 1) e a equação simplificada do balanço de energia (equação 2), foram calculados o fluxo de calor latente (IE) (equação 3) e o fluxo de calor sensível (H) (equação 4), empregando-se a razão de Bowen (b) como utilizado por Pereira et al. (1997) (equação5):

$$
\begin{gathered}
\beta=\frac{H}{\lambda E} \\
\mathrm{SR}+\mathrm{G}+\lambda \mathrm{E}+\mathrm{H} \cong 0
\end{gathered}
$$

Em que: $S R$ = saldo de radiação; $G$ = fluxo de calor para o solo.

$$
\begin{gathered}
\lambda E=-\frac{(S R+G)}{(1+\beta)} \\
\text { para } \beta \neq-1
\end{gathered}
$$

$$
\begin{aligned}
& \mathrm{H}=-(\mathrm{SR}+\lambda \mathrm{E}+\mathrm{G}) \\
& \beta=\left[\frac{\Delta T u}{(1-W) \Delta T s}-1\right]^{-1}
\end{aligned}
$$


em que, W é um fator de ponderação que depende da temperatura do bulbo úmido (Tu) e da constante psicrométrica $(\gamma)$, podendo ser calculado através das equações:

$$
\begin{aligned}
& \mathrm{W}=0,407+0,0145 \mathrm{Tu}, \quad \text { para } 0<\mathrm{Tu}<16^{\circ} \mathrm{C} \\
& \mathrm{W}=0,483+0,01 \mathrm{Tu}, \text { para } 16,1<\mathrm{Tu}<32^{\circ} \mathrm{C}
\end{aligned}
$$

propostas por Wilson e Rouse (1972) e Viswanadham et al. (1991), citados por Pereira et al. (1997).

\section{RESULTADOS E DISCUSSÃO}

Na figura 1 é apresentado um resumo das condições meteorológicas ocorridas durante o período experimental. No período de 10 a 30 de novembro de 1998 foi verificada baixa ocorrência de chuvas e temperaturas elevadas, caracterizando o período seco do experimento. No início de dezembro até o dia 15 do mesmo mês, verificaram-se chuvas intensas até de 55 mm diários, ocorrida em 10 de dezembro, caracterizando o período úmido do experimento.

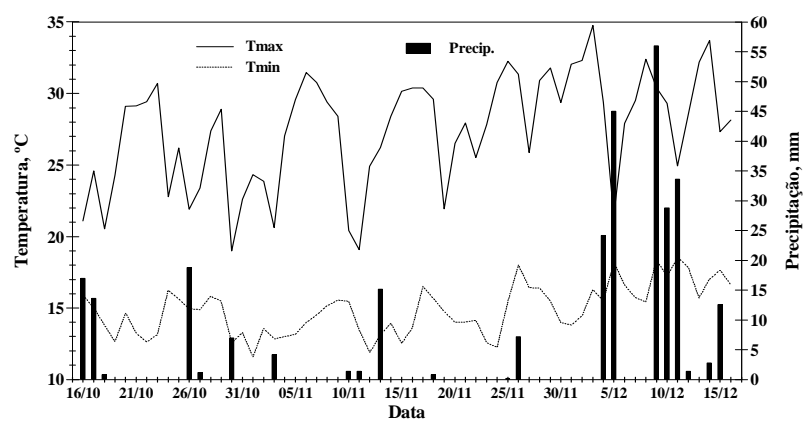

Figura 1. Variação diária das temperaturas máximas e mínimas e da precipitação pluvial de 16 de outubro a 16 de dezembro de 1998, em Jundiaí (SP).

A figura 2 apresenta a relação entre os valores diários da radiação solar global (RG) obtida em posto meteorológico e o saldo de radiação (SR) obtido na cultura da videira. Durante o período de medições, a razão entre o saldo de radiação e a radiação solar global (SR/RG) variou entre 0,54 e 0,85. A equação de regressão obtida considerando o coeficiente linear igual a zero, foi:

$$
S R=0,68 R G,
$$

com um coeficiente de determinação de 0,85 . Trabalhando com videira, cv. Itália, cultivada em latada, TEIXEIRA et al. (1997), obtiveram valores de razão SR/ RG, entre $57 \%$ e $71 \%$. Segundo esses autores, tal variação ocorreu devido ao estádio fenológico da cultura e à umidade do solo.

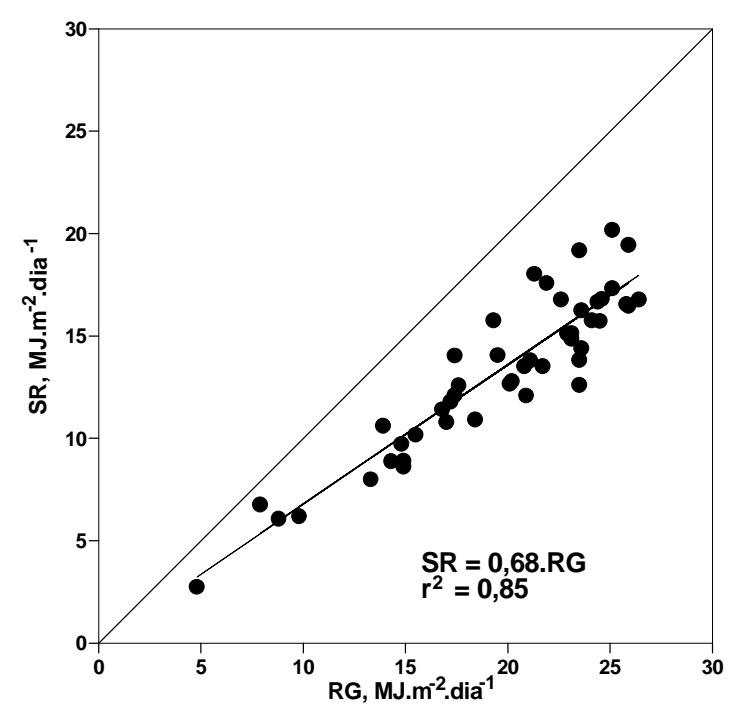

Figura 2. Relação entre valores diários de radiação solar global (RG) obtida em posto meteorológico e do saldo de radiação (SR) obtido em videira, cv. Niagara Rosada, conduzida em espaldeira, em Jundiaí (SP).

Quando a relação entre os valores de SR e RG é feita em nível horário (Figura 3), determina-se a estimativa do saldo de radiação a partir da radiação solar global pela seguinte equação:

$$
\mathrm{SR}=-45,91+0,809 R \mathrm{R},
$$

apresentando coeficiente de determinação de 0,96 . Segundo Cunha et al. (1993), coeficientes de determinação acima de 0,9 são comuns quando relacionam saldo de radiação e radiação solar global, mostrando ser boa relação para estimativa do saldo de radiação a partir de dados de radiação solar global.

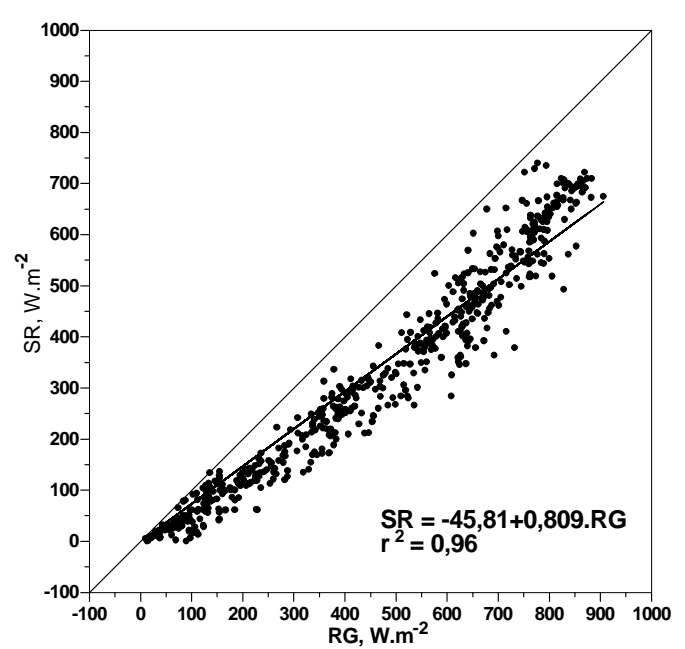

Figura 3. Relação entre os valores horários da radiação solar global (RG) obtida em posto meteorológico e do saldo de radiação (SR) obtido em videira, cv. Niagara Rosada, conduzida em espaldeira, em Jundiaí (SP). 
A figura 4 mostra a variação horária do fluxo de calor no solo, na rua mantida capinada e forrada com capim-gordura seco e sua relação com o saldo de radiação. Em 21/11/1998 (Figura 4A), caracterizado por apresentar céu limpo, o fluxo de calor no solo representou $7,5 \%$ do saldo de radiação na rua mantida capinada e 2,5\% na rua com forro. Em 11/12/1998 (Figura 4B), caracterizado como dia nublado, o fluxo de calor no solo representou $2,8 \%$ e $0,5 \%$, do saldo de radiação, respectivamente, nas ruas mantidas capinada e com forro.

Considerando-se o período de medições, o fluxo de calor no solo representou, em média, 5,7\% e 1,3\% do saldo de radiação para as ruas mantidas capinada e com forro respectivamente. No caso das ruas mantidas capinadas, o sentido do fluxo observado foi da comunidade vegetal para o solo, enquanto o valor médio do fluxo obtido para as ruas forradas foi no sentido inverso, ou seja, do solo para a comunidade de plantas.

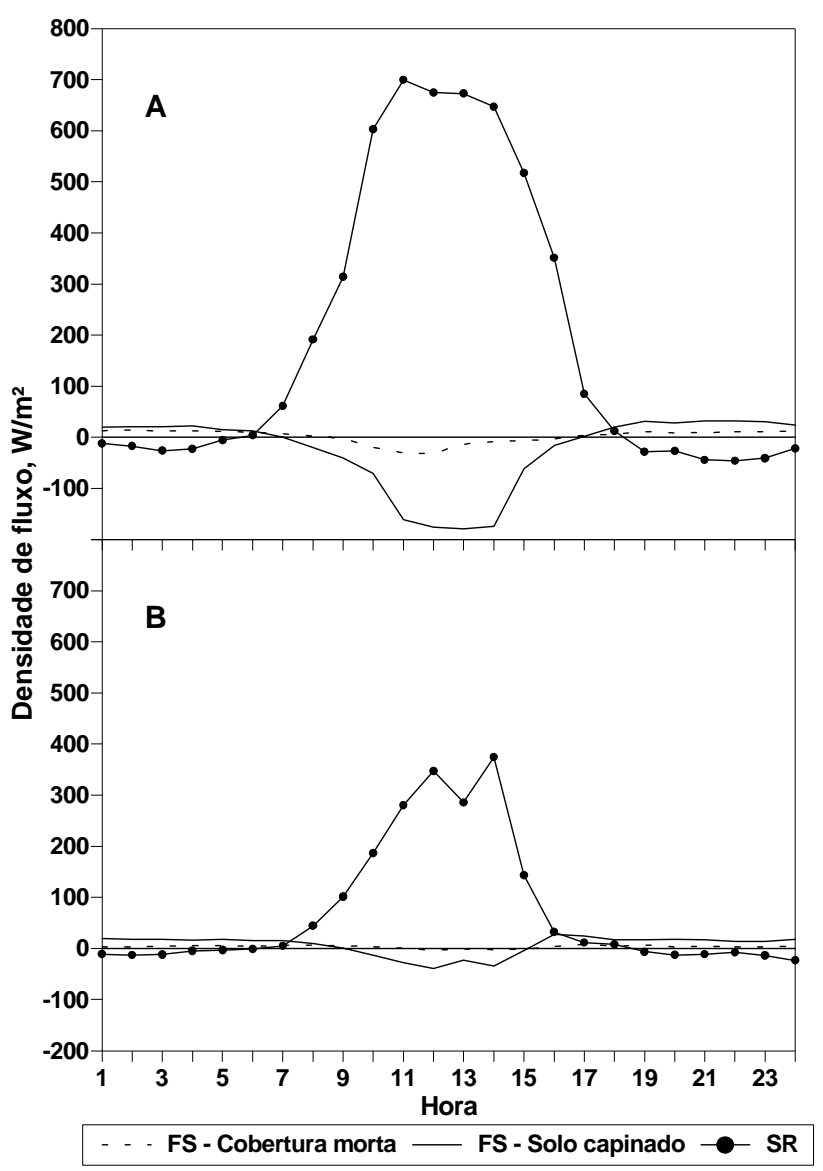

Figura 4. Variação horária do saldo de radiação (SR) e do fluxo de calor no solo (FS) nos dias 21/11/1998 (Céu limpo - A) e 11/12/1998 (Nublado - B) obtidos em videira, cv. Niagara Rosada, conduzida em espaldeira, em Jundiaí (SP).
A partição da energia disponível ao sistema (SR) nos fluxos de calor latente (LE), de calor sensível $(H)$ e de calor no solo $(G)$ varia de acordo com as condições atmosféricas, de umidade do solo e de desenvolvimento da cultura (Pereira et al., 1997). Para caracterizar o balanço de energia em vinhedo em diferentes condições atmosféricas são apresentados os resultados da variação horária de seus componentes em videira Niagara Rosada na figura 5.

O dia 25/11/1998 (Figura 5A) foi característico do período seco, ocorrido durante o experimento, com elevada incidência de energia $(\mathrm{SR}=16,81$ $\mathrm{MJ} . \mathrm{m}^{-2} \cdot$ dia $^{-1}$, de saldo de radiação) e 12 dias após a ocorrência da última chuva significativa (Figura 1).

Os valores de fluxo de calor no solo utilizados no cálculo do balanço de energia foram obtidos com a média dos valores obtidos nos fluxímetros instalados na rua capinada e na rua forrada com capim gordura seco.

Pode-se verificar que o fluxo de calor sensível representou aproximadamente $48 \%$ do saldo de radiação nesse dia, e supera o fluxo de calor latente, que foi de $44 \%$ do SR, a partir das 11 horas, com valor de $\beta$ atingindo o máximo às 15 horas $(4,18)$. Oliver e SENE (1992), em estudos de balanço de energia em vinhedos, em implantação no Sudoeste da Espanha, durante o período seco, obtiveram valores médios de $\beta$ igual a 2, no período vespertino, enquanto em terra arada esses valores chegaram a 7 .

A partir das 16 horas, com a diminuição do suprimento de energia os valores de $\beta$ voltaram a decrescer. Nesse dia, o fluxo de calor no solo representou 9\% do saldo de radiação. O dia 11/12/1998 (Figura 5B) representa a partição da energia disponível $(6,04$ MJ.m ${ }^{-2} \cdot$ dia $^{-1}$ ) em um dia chuvoso. Nesse dia, o fluxo de calor latente $\left(5,17 \mathrm{MJ} \cdot \mathrm{m}^{-2} \cdot \mathrm{dia}^{-1}\right)$ representou $86 \%$ do saldo de radiação, enquanto o fluxo de calor sensível $\left(1,32 \mathrm{MJ} \cdot \mathrm{m}^{-2} \cdot \mathrm{dia}^{-1}\right)$ representou $21 \%$.

Devido à ocorrência de chuva nesse dia e em dias anteriores (Figura 1), o solo estava em processo de resfriamento e o fluxo apresentou, em boa parte do dia, valores positivos, representando um incremento de energia para a superfície de $7 \%$ do saldo de radiação.

O dia 13/12/1998 (Figura 5C) apresenta a variação horária dos componentes do balanço de energia em um dia ensolarado, após um período de chuva. Pode-se observar que os valores do saldo de radiação se mantiveram entre 600 e $700 \mathrm{~W} . \mathrm{m}^{-2}$ durante o período que foi das 10 às 15 horas, havendo uma redução a partir das 16 horas, causando uma diminuição considerável em todos os componentes do balanço. 
Nesse dia, maior parte do saldo de radiação foi destinada ao fluxo de calor latente $(68 \%)$, seguido do fluxo de calor sensível (29\%) e do fluxo de calor para o solo (3\%); os valores de $\beta$ se mantiveram entre 0,3 e 0,7 durante o período diurno.

Analisando-se a variação dos componentes do balanço de energia na cultura durante 20 dias ao longo do experimento (Tabela 1), pode-se verificar que a partição do SR em fluxo de calor latente (LE), variou de um mínimo de $40 \%$ até um máximo de $86 \%$, com média de 59\%. TEIXEIRA et al. (1997), em estudo com videira Itália cultivada em latada, sob irrigação, obti- veram partição de SR em LE com variações entre $55 \%$ e $105 \%$, com média de $82 \%$. Os valores de LE/SR acima de $100 \%$ verificado pelos autores, se devem ao efeito de advecção de calor sensível na cultura, conduzido sob irrigação. Nas condições experimentais de Jundiaí, como não havia irrigação, o fluxo de calor sensível $(\mathrm{H})$ no período analisado variou de $21 \%$ a $56 \%$, com média de $38 \%$, não havendo razão H/SR positiva em nenhum dos dias analisados. O fluxo de calor no solo (FS), obtido pela média das ruas mantidas capinadas e forradas, apresentou valor médio de sua relação com SR de 3\%.
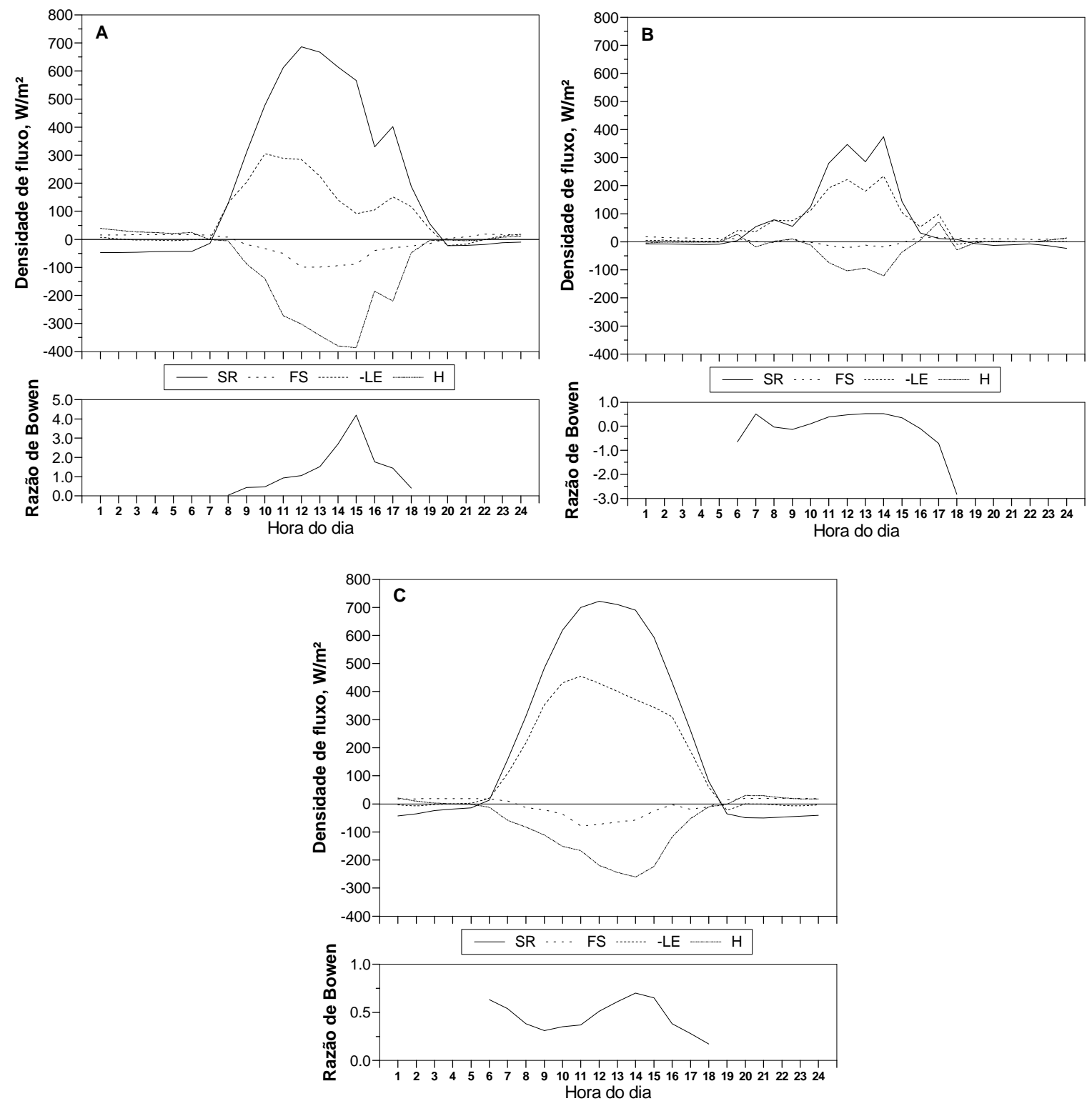

Figura 5. Variação horária do saldo de radiação (SR), do fluxo de calor no solo (FS), do fluxo de calor latente (LE), do fluxo de calor sensível $(\mathrm{H})$ e da razão de Bowen nos dias 25/11/1998 (Período seco - A), 11/12/1998 (Dia nublado B) e 1312/1998 (Dia ensolarado - C), em videira Niagara Rosada, no município de Jundiaí (SP). 
Quadro 1. Variação diária dos valores do saldo de radiação (RS), fluxo de calor latente (LE), fluxo de calor sensível (H) e fluxo de calor no solo (FS) (MJ.m . dia $^{-1}$ ) e relações de LE, H e FS com RS, durante o período de 15/11 a 15/12/1998 em videira Niagara Rosada, no município de Jundiaí (SP)

\begin{tabular}{|c|c|c|c|c|c|c|c|}
\hline Dia & SR & $\mathrm{LE}$ & $\mathrm{H}$ & FS & LE/SR & H/SR & FS/SR \\
\hline & \multicolumn{4}{|c|}{ MJ.m $\mathrm{m}^{-2} \cdot \mathrm{dia}^{-1}$} & & & \\
\hline $15 / 11$ & 16,79 & $-10,24$ & $-5,94$ & $-0,62$ & $-0,60$ & $-0,37$ & $-0,03$ \\
\hline $16 / 11$ & 15,17 & $-7,14$ & $-7,16$ & $-0,87$ & $-0,47$ & $-0,47$ & $-0,06$ \\
\hline $17 / 11$ & 12,78 & $-7,40$ & $-4,44$ & $-0,94$ & $-0,58$ & $-0,35$ & $-0,07$ \\
\hline $18 / 11$ & 10,55 & $-6,86$ & $-3,62$ & $-0,07$ & $-0,65$ & $-0,34$ & $-0,01$ \\
\hline $19 / 11$ & 10,83 & $-5,58$ & $-5,51$ & 0,25 & $-0,51$ & $-0,51$ & 0,02 \\
\hline $20 / 11$ & 12,03 & $-7,32$ & $-4,30$ & $-0,40$ & $-0,61$ & $-0,36$ & $-0,03$ \\
\hline $21 / 11$ & 16,35 & $-8,64$ & $-6,73$ & $-0,98$ & $-0,53$ & $-0,41$ & $-0,06$ \\
\hline $22 / 11$ & 16,32 & $-6,59$ & $-9,09$ & $-0,64$ & $-0,40$ & $-0,56$ & $-0,04$ \\
\hline $23 / 11$ & 13,85 & $-6,65$ & $-6,70$ & $-0,50$ & $-0,48$ & $-0,48$ & $-0,04$ \\
\hline $24 / 11$ & 15,82 & $-8,97$ & $-5,70$ & $-1,15$ & $-0,57$ & $-0,36$ & $-0,07$ \\
\hline $25 / 11$ & 16,94 & $-7,42$ & $-8,05$ & $-1,47$ & $-0,44$ & $-0,48$ & $-0,09$ \\
\hline $06 / 12$ & 11,25 & $-7,37$ & $-3,88$ & $-0,01$ & $-0,65$ & $-0,34$ & 0,00 \\
\hline $07 / 12$ & 15,64 & $-11,36$ & $-4,06$ & $-0,23$ & $-0,73$ & $-0,26$ & $-0,01$ \\
\hline 08/12 & 17,23 & $-8,02$ & $-8,37$ & $-0,83$ & $-0,47$ & $-0,49$ & $-0,05$ \\
\hline $09 / 12$ & 11,78 & $-6,39$ & $-5,23$ & $-0,15$ & $-0,54$ & $-0,44$ & $-0,01$ \\
\hline $10 / 12$ & 12,55 & $-8,77$ & $-3,67$ & $-0,10$ & $-0,70$ & $-0,29$ & $-0,01$ \\
\hline $11 / 12$ & 6,04 & $-5,17$ & $-1,32$ & 0,45 & $-0,86$ & $-0,22$ & 0,07 \\
\hline $12 / 12$ & 10,03 & $-7,87$ & $-2,07$ & $-0,09$ & $-0,79$ & $-0,21$ & $-0,01$ \\
\hline $13 / 12$ & 19,34 & $-13,09$ & $-5,64$ & $-0,61$ & $-0,68$ & $-0,29$ & $-0,03$ \\
\hline $14 / 12$ & 14,01 & $-9,30$ & $-4,30$ & $-0,40$ & $-0,66$ & $-0,31$ & $-0,03$ \\
\hline $15 / 12$ & 8,81 & $-6,45$ & $-2,63$ & 0,27 & $-0,73$ & $-0,30$ & 0,03 \\
\hline Média & 13,53 & $-7,93$ & $-5,16$ & $-0,43$ & $-0,59$ & $-0,38$ & $-0,03$ \\
\hline
\end{tabular}

Valores negativos indicam fluxos para fora da comunidade de plantas.

\section{CONCLUSÕES}

1. O saldo de radiação (SR) sobre o vinhedo de 'Niagara Rosada' conduzida em espaldeira pode ser estimado a partir dos valores de radiação solar global incidente.

2. A maior parte do saldo de radiação (SR) sobre o vinhedo de 'Niagara Rosada' é utilizada como fluxo de calor latente (59\%), com variação de $40 \%$ a $86 \%$, seguido de fluxo de calor sensível $(38 \%)$, com variação de $21 \%$ a $56 \%$ e fluxo de calor no solo (3\%), com variação de $1 \%$ a $9 \%$.

\section{REFERÊNCIAS BIBLIOGRÁFICAS}

ALVES, A.V.; AZEVEDO, P.V.; SILVA, B.B. Balanço de energia e reflectância de um cultivo de melão. Revista Brasileira de Agrometeorologia, Santa Maria, v.6, n.2, p.139-146, 1998.

CUNHA, G.R.; PAULA, J.R.F.; BERGAMASCHI, H.; SAIBRO, J.C.; BERLATO, M.A. Balanço de radiação em alfafa. Revista Brasileira de Agrometeorologia, Santa Maria, v.1, p. 1-10, 1993

CUNHA, G.R.; BERGAMASCHI, H., BERLATO, M.A., MATZENAUER, R. Balanço de energia em cultura de milho. Revista Brasileira de Agrometeorologia, Santa Maria, v.4, n.1, p.1-14, 1996. 
HEILMAN, J.L.; McINNES, K.J.; SAVAGE, M.J. et al. Soil and canopy energy balances in a west Texas vineyard. Agricultural and Forest Meteorology, Amsterdam, v. 71, n.1, p. 99-114, 1994.

LOPES, P.M.O.; SILVA, B.B.; AZEVEDO, P.V. et al. Balanço de energia num pomar de mangueiras irrigado. Revista Brasileira de Agrometeorologia, Santa Maria, v. 9, n.1, p.1-8, 2001.

PEREIRA, A.R.; VILLA NOVA, N.A.; SEDYAMA, G.C. Evapotranspiração. Piracicaba: FEALQ, 1997. 183p.

POMMER, C.V.; PASSOS, I.R.S. Fisiologia da videira: como produz açúcar uma videira? Campinas: Instituto Agronômico, 1990. 20p. (Documentos IAC, 20)

POMMER, C.V.; PEDRO JUNIOR, M.J., MARONI, L.G., PIRES, E.J.P., TERRA, M.M., MARTINS, F.P., PASSOS, I.P.S. Alternativas para cobertura morta em videira. Revista Brasileira de Fruticultura, Cruz das Almas, v.13, n.4, p.217-225, 1991.
VILLA NOVA, N.A.; PEREIRA, A.R.; PEDRO JÚNIOR, M.J. Balanço de energia numa cultura de arroz em condições de sequeiro. Bragantia, Campinas, v. 34, n.9, p 171-176, 1975.

OLIVER, H.R.; SENE, K.J. Energy and water balances of developing vines. Agricultural and Foret Meteorology, Amsterdam, v. 61, n.2, p. 167-185, 1992.

SMART, R.C. Sunlight interception by vineyards. American Journal Enology Viticulture, Davis, v.24, p.141-147, 1973.

TEIXEIRA, A.H.C.; AZEVEDO, P.V.; SILVA, B.B.; SOARES, J.M. Balanço de energia na cultura da videira, cv. "Itália". Revista Brasileira de Agrometeorologia, Santa Maria, v.5, n.2, p.137-141, 1997. 